\title{
Palmarplantar Keratodermas
}

Melissa Gitman, $M D, C M$

\section{Case Presentation}

A 44 year old African American female with a history of seizure disorder and cirrhosis secondary to alcohol abuse was brought to the emergency department (ED) after a witnessed generalized tonic-clonic seizure on the street. While the patient was in the ED, she was observed to have $500 \mathrm{cc}$ of bright red hemetemesis and promptly transferred to the intensive care unit with successful stabilization of an upper GI bleed. It was not until transfer back to the telemetry that she was noted to have a shiny, scaly lesions on her hands and feet bilaterally (Figures 1 and 2).

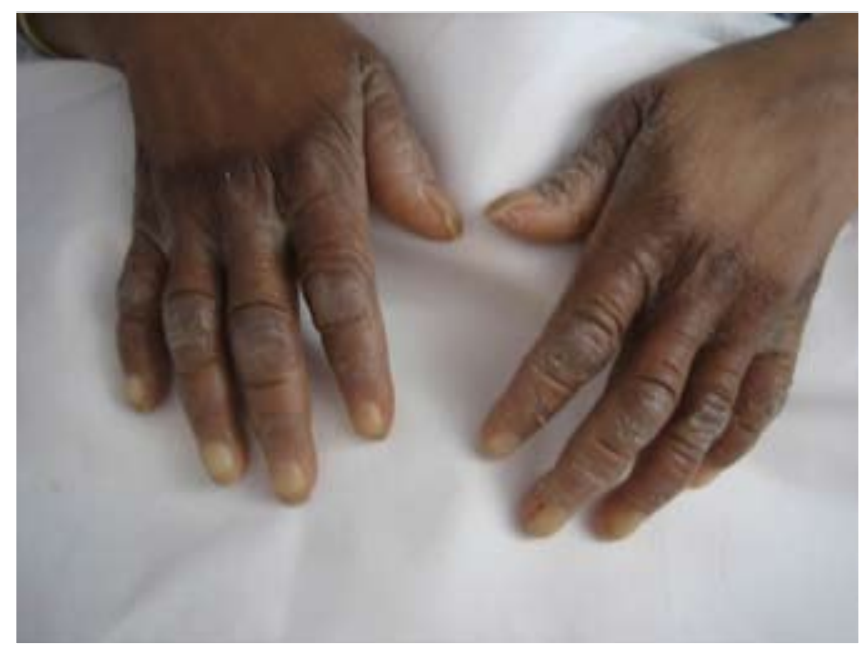

Figure 1.

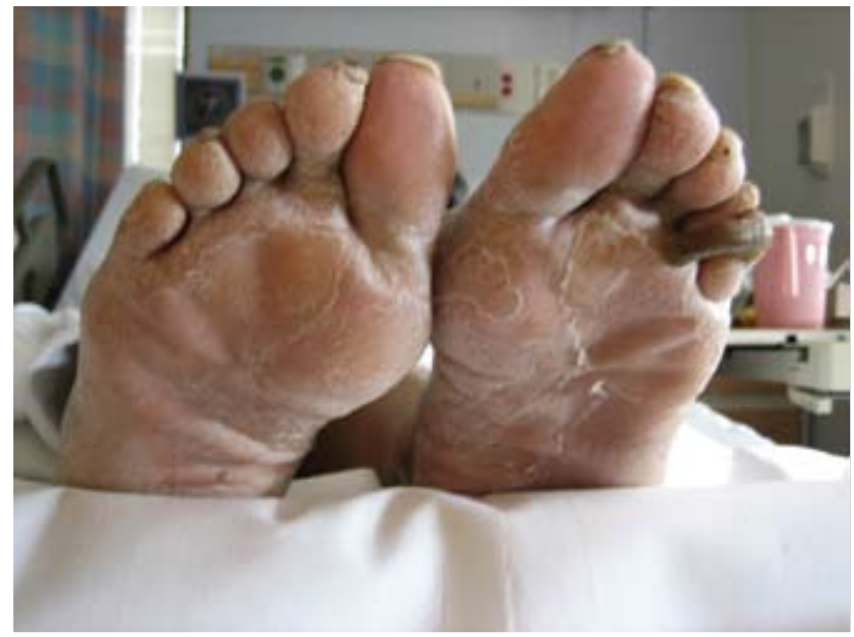

Figure 2.

\section{Discussion}

When evaluating a patient with PPK, the first step is to perform a detailed history and physical, including a complete skin exam. Patel et al proposed an algorithm to evaluate patients thought to have acquired PPK of unknown etiology (Figure 3 ). ${ }^{1}$

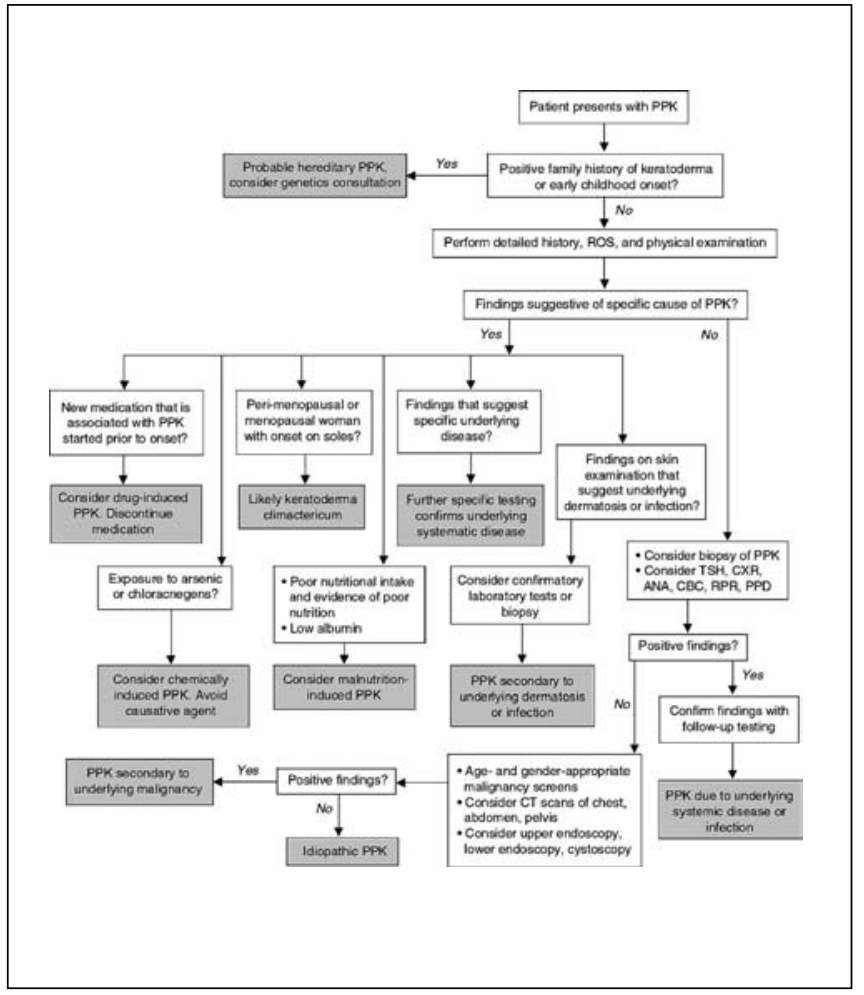

Figure 3. Adapted from Patel et al

Palmoplantar keratodermas (PPK) is comprised of a heterogeneous group of conditions that are characterized by hyperkeratosis of the palms and soles. ${ }^{1}$ These conditions can be classified in several different fashions. First of all, a distinction between the hereditary and acquired forms must be determined. Furthermore the hereditary forms can be further subdivided depending on the extent of involvement of the epidermis (focal, diffuse or punctuate), the location of the lesions, the mode of inheritance, the age of onset and the presence of comorbid conditions. Moreover, with advancements in molecular genetics, these conditions can also be classified by the type of gene dysfunction that occurs, including mutations in the genes responsible for synthesis of structural proteins, cornified envelope, cohesion, connexins and trans-membrane signal transducers. ${ }^{2}$ While PPK may be found in the absence of other conditions, it is often found as part of a disease complex, such as skin findings associated with deafness, corneal dystrophy, cardiac structural abnormalities, gingival hyperplasia and esophageal malignancy. In the hereditary form, a genetics consult should be obtained in order to further evaluate the specific type of PPK present. ${ }^{2}$

Similarly the acquired form of the condition can be further subdivided into categories based on their etiology. Patel et al proposed the acquired PPK's be subdivided as follows: 
keratoderma climactericum, drug related, malnutrition associated, chemically induced, systemic disease related, malignancy associated, dermatoses related, infectious etiology and idiopathic. ${ }^{1}$ Keratoderma climactericum is typically found in menopausal women, frequently with co-existent obesity or hypertension. The condition begins with involvement of the plantar aspect of the feet, where the patient develops erythema, hyperkeratosis and fissuring, leading to difficulty ambulating. Palmar involvement occurs later in the disease course. Chemical associated PPK has been described with a history of exposure to arsenic and chloracnegens. Symptoms generally resolve a few months after removal of the exposure, however, in some cases, symptoms have persisted for several years. Severe malnutrition in concentration camp inmates has also been reported to be associated with acquired PPK. A deficiency in both protein and vitamins led to the condition which improved with vitamin replacement. Multiple medications, including glucan, lithium, venlafaxine, verapamil, quinacrine and certain chemotherapy agents, have all had case reports in the literature associating their use with PPK. Generally, the diagnosis is made in retrospect with resolution of the symptoms upon discontinuation of the offending agent. Several dermatologic conditions, including psoriasis, lichen planus and eczema have all been reported to cause a reactive form of $\mathrm{PPK}$. Certain infections, including HIV, syphilis, scabies and TB have all been reported to cause hyperkeratosis. PPK is also considered to be a paraneoplastic marker for malignancies of the esophagus, lung, breast, blood, bladder, and GI tract.

Tripe palm is a specific finding which refers to form of palmar keratoderma with a velvet-like texture and exaggeration of the lines of the palm and fingers. Greater than $90 \%$ of patients with this finding have an underlying malignancy. Of course, idiopathic acquired PPK is a diagnosis of exclusion after all other etiologies have been eliminated. ${ }^{1}$

Treatment of PPK usually involves treatment of the underlying etiology. If no causative process is identified then a conservative approach is undertaken using topical therapies to lessen the dermatologic symptoms. ${ }^{1}$

\section{References}

1. Patel S, Zirwas M, English JC 3rd. Acquired palmoplantar keratoderma. Am J Clin Dermatol 2007;8:1-11.

2. Itin PH, Fistarol SK. Palmoplantar keratodermas. Clin Dermatol 2005;23:15-22. 